\title{
Mock-Chebyshev Polinom İnterpolasyonu
}

\author{
B. Ali İbrahimoğlu ${ }^{1 *}$ \\ 1*Y Yıldız Teknik Üniversitesi, Kimya-Metalürji Fakültesi, Matematik Mühendisliği Bölümü, İstanbul, Türkiye (ORCID: 0000-0002-3644-2201), \\ bibrahim@yildiz.edu.tr
}

(İlk Geliş Tarihi 16 Eylül 2021 ve Kabul Tarihi 30 Kasım 2021)

(DOI: $10.31590 /$ ejosat.996547)

ATIF/REFERENCE: İbrahimoğlu, B. A. (2021). Mock-Chebyshev Polinom İnterpolasyonu. Avrupa Bilim ve Teknoloji Dergisi, (27), 858-865.

$\ddot{O} \mathbf{z}$

Polinom interpolasyonunda noktaların geometrik yeri önemli bir rol oynar. Bunu göstermek için, iyi bilinen Runge fenomenini örnek vermek yeterli olacaktır. Bu bağlamda, noktalarının dağılımı eşit aralıklı olmaktan epey uzak olan Chebyshev nodlarının kullanılması tavsiye edilir. Fakat, bir gözlem veya ölçüm sırasında veriler genelde eşit aralıklı olacak şekilde elde edilir. Bu eşit aralıklı verileri kullanarak polinom interpolasyonu ile bir yaklaşım elde edilmek istendiğinde hem Runge olgusundan kaçınmak hem de Chebyshev noktalarının kullanılmasına benzer şekilde iyi bir sonuç elde etmek için takip edilebilecek en iyi stratejilerden biri son zamanlarda geliştirilen mock-Chebyshev noktalarını kullanmaktır. Eşit aralıklı noktaların genişçe bir kümesinden seçilerek elde edilen bu noktalar asimptotik olarak Chebyshev noktalarının dağılımını takip eder. Ancak, bu noktaların hesaplanması için gereken işlem yükü fazladır ve bu konuda literatürde yeteri kadar çalışma yoktur. Bu çalışmada, mock-Chebyshev noktalarının elde edilmesinde düşük işlem yüküne sahip iyi bir alternatif bir yöntem sunulmaktadır. Dahası, bu yöntem mock-Chebyshev noktalarının birbirinden farklı kümelerinin elde edilmesini sağlar. Kovid-19 vakalarını tahmin etmek için bu metod ile elde edilen noktalar kullanılarak polinom interpolasyonun bir uygulaması verilmiştir.

Anahtar Kelimeler: Polinom interpolasyonu, Mock-Chebyshev noktaları, Nod dağılımları.

\section{Mock-Chebyshev Polynomial Interpolation}

\begin{abstract}
In polynomial interpolation, the location of the interpolation points plays an important role. Suffices to consider the well-known Runge phenomenon to understand this. In this context, the general recommendation is to use the highly non-uniform Chebyshev nodes. But, during an observation or measurement, the data are generally obtained for the equispaced points. When it is aimed to obtain an approximation with polynomial interpolation using equispaced nodes, one of the best strategies has been the use of the lately developed mock-Chebyshev points in order to avoid the Runge phenomenon and to get similarly better results with the use of Chebyshev points. A disadvantage here is that the computational cost is higher and there are not many studies in the existing literature. In this study, a better alternative method is introduced for constructing a set of mock-Chebyshev interpolation points with a low computational cost. Moreover, using this method, it is also possible to obtain different configurations of mock-Chebyshev sets. Using the points obtained by the method, an application of polynomial interpolation is provided in order to estimate Covid-19 cases.
\end{abstract}

Keywords: Polynomial interpolation, Mock-Chebyshev points, Node distributions.

\footnotetext{
*Sorumlu Yazar: bibrahim@yildiz.edu.tr
} 


\section{Giriş}

Polinom interpolasyonunda noktaların dağılımı önemli bir rol oynar. Bazı nokta kümeleri ile optimale (en iyiye) yakın yaklaşımlar elde edilirken, bazılarıyla çok kötü (ıraksak) sonuçlar elde edilebilir. Bunu göstermek için, interpolasyonda eşit aralıklı noktaları ve noktalarının dağılımı eşit aralıklı olmaktan epey uzak olan Chebyshev noktalarını ele almak yeterlidir. Eşit aralıklı noktalar kullanıldığında sürekli fonksiyonların çoğu için dahi interpolasyon polinomu düzgün yakınsamaz. Buna standart örnek olarak, $[-1,1]$ aralığında $f(x)=\left(1+25 x^{2}\right)^{-1}$ Runge fonksiyonunun polinom interpolasyonu verilmektedir. Bu fonksiyon için interpolasyon polinomu eşit aralıklı noktalar kullanılarak oluşturuluyorsa interpolasyon aralığının sınırlarına doğru gidildikçe çok büyük salınımlar görülecek ve böylece interpolasyon polinomu Runge fonksiyonuna yakınsamayacaktır [7]. Bu durum Runge olgusu olarak adlandırılır.

Buna karşın, interpolasyon aralığının sınırlarına doğru gidildikçe önemli ölçüde fazla noktanın mevcut olduğu ve $[-1,1]$ aralığı için bu noktaların dağııımının $n\left(\pi \sqrt{1-x^{2}}\right)^{-1}$ yoğunluk fonksiyonuna sahip olduğu Chebyshev noktalarında ise durum tamamen farklıdır. Örneğin, Runge fonksiyonu için Chebyshev noktalarında interpolasyon polinomu geometrik bir şekilde yakınsar. Dahası, tüm analitik fonksiyonlar için bu durum geçerlidir.

Ancak, pek çok bilimsel araştırmada veriler genelde eşit aralıklı noktalarda elde edilir. Örneğin, bir deney veya gözlemde ölçümler genellikle eşit aralıklı olacak şekilde yapılır. Bu eşit aralıklı verileri kullanarak polinom interpolasyonu ile bir yaklaşım elde edilmek istendiğinde, hem Runge olgusundan kaçınmak hem de Chebyshev noktalarının kullanılmasına benzer şekilde iyi bir sonuç elde etmek için takip edilebilecek en iyi stratejilerden biri son zamanlarda geliştirilen mock-Chebyshev noktalarını kullanmaktır [1]. Eșit aralıklı noktaların yeterince genişçe bir kümesinden seçilerek elde edilen bu noktalar asimptotik olarak Chebyshev noktalarının dağılımını takip ederler. Ancak, bu noktaların hesaplanması için gereken işlem yükü fazladır ve bu konuda literatürde yeteri kadar çalıșma yoktur.

Runge olgusunu önleyen bir yöntem olarak, polinom interpolasyonunda mock-Chebyshev noktalarının kullanılması ve bu noktaların nasıl seçileceği konusunda bir strateji ilk kez Boyd tarafindan verilmiştir [1]. Ancak, belirtilen yöntem $O\left(n^{3}\right)$ mertebeden işlem yükününe sahip olması nedeniyle, noktaların hesaplanması yüksek dereceden yaklaşımlar için zor olacaktır. Bir başka çalışmada ise eşit aralıklı noktaların sayısının serbestçe seçilebildiği durum için mock-Chebysev noktalarını hesaplayan hızı ıir algoritma $[4,5]$ verilmiştir. Yakın zamanda yayınlanan ve mock-Chebyshev noktalarının özelliklerinin çalışmadaki [6] öneri dikkate alınarak mockChebyshev noktalarını $O\left(n^{2}\right)$ mertebeden işlem yükü ile hesaplayan bir yöntem bu çalışmada verilmiştir. Bu yöntem ile elde edilen noktaların performansını test etmek için bu noktalar polinom interpolasyonunda kullanılmıştır. Bunun için Kovid-19 vakalarını ara günlerde tahmin eden interpolasyon polinomları elde edilmiştir. Elde edilen sonuçlar, eşit aralıklı noktalar kullanılması durumunda elde edilen sonuçlar ile karşıllaştırılmıştır.

\section{Polinom İnterpolasyonu}

Birbirinden farklı $x_{0}, \ldots, x_{n} \in[a, b] \subset \mathbb{R}$ noktaları (veya nodları) ve bu noktalardaki $f_{0}, \ldots, f_{n}$ verileri (veya fonksiyon değerleri) kullanılarak,

$$
p_{n}\left(x_{j}\right)=f_{j}, \quad j=0, \ldots, n
$$

interpolasyon şartlarını sağlayan ve derecesi en fazla $n$ olan tek bir interpolasyon polinomu $p_{n}(x)$ vardır. $\mathrm{Bu} p_{n}(x)$ polinomu çeşitli polinom bazları kullanılarak farklı formlarda ifade edilebilir. Baz seçimi matematiksel açıdan bir fark oluşturmasa da interpolasyon polinomunu nümerik olarak hesaplarken problemin durumunu etkiler.

\subsection{Monomial Bazların Kullanılması}

Denklem (1)'deki interpolasyon şartlarını sağlayan $p_{n}(x)$ polinomu $1, x, \ldots, x^{n}$ monomial bazlarının bir lineer kombinasyonu şeklinde aşağıdaki gibi ifade edilebilir: $p_{n}(x)=a_{0}+a_{1} x+\cdots+a_{n-1} x^{n-1}+a_{n} x^{n}=\sum_{i=0}^{n} a_{i} x^{i}$.

Burada oluşan $a_{0}, \ldots, a_{n}$ katsayıları, Denklem (1)'deki interpolasyon şartları kullanılarak

$$
\left[\begin{array}{ccccc}
1 & x_{0} & x_{0}^{2} & \cdots & x_{0}^{n} \\
1 & x_{1} & x_{1}^{2} & \cdots & x_{1}^{n} \\
1 & x_{2} & x_{2}^{2} & \cdots & x_{2}^{n} \\
\vdots & \vdots & \vdots & \ddots & \vdots \\
1 & x_{n} & x_{n}^{2} & \cdots & x_{n}^{n}
\end{array}\right]\left[\begin{array}{c}
a_{0} \\
a_{1} \\
a_{2} \\
\vdots \\
a_{n}
\end{array}\right]=\left[\begin{array}{c}
f_{0} \\
f_{1} \\
f_{2} \\
\vdots \\
f_{n}
\end{array}\right]
$$

şeklinde Vandermonde sistemi olarak adlandırılan ve $V \mathbf{a}=\mathbf{f}$ şeklinde gösterilecek $n+1$ boyutlu bir lineer denklem sisteminin çözümü ile hesaplanabilir. Burada oluşan $(n+1) \times(n+1)$ boyutundaki kare matris, katsayılar matrisidir. Bu katsayılar matrisine Vandermonde matrisi denir ve aşağıdaki şekilde gösterilir: 


$$
V=V_{n}\left(x_{0}, \ldots, x_{n}\right)=\left[\begin{array}{ccccc}
1 & x_{0} & x_{0}^{2} & \cdots & x_{0}^{n} \\
1 & x_{1} & x_{1}^{2} & \cdots & x_{1}^{n} \\
1 & x_{2} & x_{2}^{2} & \cdots & x_{2}^{n} \\
\vdots & \vdots & \vdots & \ddots & \vdots \\
1 & x_{n} & x_{n}^{2} & \cdots & x_{n}^{n}
\end{array}\right] .
$$

Burada oluşan $V \mathbf{a}=\mathbf{f}$ lineer denklem sistemi ancak ve ancak $\operatorname{det} V \neq 0$ ise, tek bir çözüme sahiptir. Birbirinden farklı $x_{0}, x_{1}, \ldots, x_{n}$ sayıları için Vandermonde matrisinin determinantı,

$$
\begin{aligned}
\operatorname{det} V_{n}\left(x_{0}, \ldots, x_{n}\right) & =\operatorname{det}\left[\begin{array}{ccccc}
1 & x_{0} & x_{0}^{2} & \cdots & x_{0}^{n} \\
1 & x_{1} & x_{1}^{2} & \cdots & x_{1}^{n} \\
1 & x_{2} & x_{2}^{2} & \cdots & x_{2}^{n} \\
\vdots & \vdots & \vdots & \ddots & \vdots \\
1 & x_{n} & x_{n}^{2} & \cdots & x_{n}^{n}
\end{array}\right] \\
& =\prod_{0 \leq j \leq n}\left(x_{i}-x_{j}\right)
\end{aligned}
$$

eşitliğini sağlar. Burada, $x_{0}, x_{1}, \ldots, x_{n}$ sayıları birbirinden farklı olduğu sürece, $\operatorname{det} V \neq 0$ 'dır. Böylece, $f_{0}, \ldots, f_{n}$ verilerinin herhangi bir seçimi için $V \mathbf{a}=\mathbf{f}$ lineer denklem sisteminin her zaman tek bir çözümü vardır.

\subsection{Lagrange Bazlarının Kullanılması}

Denklem (1)'deki interpolasyon şartlarını sağlayan $p_{n}(x)$ polinomu,

$l_{i}(x)=\prod_{\substack{j=0 \\ j \neq i}}^{n} \frac{x-x_{j}}{x_{i}-x_{j}}, \quad i=0, \ldots, n$

formülü ile verilen $l_{0}(x), l_{1}(x), \ldots, l_{n}(x)$ Lagrange bazları

kullanılarak farklı bir şekilde ifade edilebilir. Burada, $i \neq j$ için $l_{i}\left(x_{j}\right)=0$ ve $i=j$ için $l_{i}\left(x_{i}\right)=1$ olduğundan $p_{n}(x)$ interpolasyon polinomu, $l_{0}(x), l_{1}(x), \ldots, l_{n}(x) \quad$ Lagrange bazlarının bir lineer kombinasyonu şeklinde aşağıdaki gibi ifade edilebilir:

$p_{n}(x)=\sum_{i=0}^{n} f\left(x_{i}\right) l_{i}(x)$.

Buna interpolasyon polinomunun Lagrange formu veya Lagrange interpolasyon polinomu denir. Burada $i$-inci Lagrange polinomu, $l_{i}(x)$ Vandermonde matrisi kullanılarak
$l_{i}(x)=\frac{\operatorname{det}\left(V_{i}(x)\right)}{\operatorname{det}(V)}=\frac{\operatorname{det} V_{n}\left(x_{0}, \ldots, x_{i-1}, x, x_{i+1}, \ldots, x_{n}\right)}{\operatorname{det} V_{n}\left(x_{0}, \ldots, x_{n}\right)}$

şeklinde ifade edilebilir. Örneğin, $n=3$ için $l_{2}(x)$ Lagrangg) polinomu

$l_{2}(x)=\frac{\operatorname{det} V_{3}\left(x_{0}, x_{1}, x, x_{3}\right)}{\operatorname{det} V_{3}\left(x_{0}, \ldots, x_{3}\right)}$

$$
=\frac{\operatorname{det}\left[\begin{array}{cccc}
1 & x_{0} & x_{0}^{2} & x_{0}^{3} \\
1 & x_{1} & x_{1}^{2} & x_{1}^{3} \\
1 & x & x^{2} & x^{3} \\
1 & x_{3} & x_{3}^{2} & x_{3}^{3}
\end{array}\right]}{\operatorname{det}\left[\begin{array}{llll}
1 & x_{0} & x_{0}^{2} & x_{0}^{3} \\
1 & x_{1} & x_{1}^{2} & x_{1}^{3} \\
1 & x_{2} & x_{2}^{2} & x_{2}^{3} \\
1 & x_{3} & x_{3}^{2} & x_{3}^{3}
\end{array}\right]}
$$

şeklinde elde edilir. Burada, $j \neq 2$ için $l_{2}\left(x_{j}\right)=0$ ve $j=2$ için $l_{2}\left(x_{2}\right)=1$ olduğu kolayca görülebilir.

\subsection{Polinom İnterpolasyonunda Hata}

Birbirinden farklı $x_{0}, x_{1}, \ldots, x_{n} \in[a, b]$ noktalarında bir $f(x)$ fonksiyonunu interpole eden ve derecesi en fazla $n$ olan interpolasyon polinomu $p_{n}(x)$ olsun. $\mathrm{Bu}$ durumda, $f(x) \in C^{(n+1)}([a, b])$ ise, oluşan hata

$$
f(x)-p_{n}(x)=\frac{f^{(n+1)}\left(\xi_{x}\right)}{(n+1) !} \prod_{j=0}^{n}\left(x-x_{j}\right)
$$

şeklinde ifade edilir. Burada, $\xi_{x} \in\left(x_{0}, x_{n}\right)$ bir sayı, $f^{(n+1)}\left(\xi_{x}\right)$ ise $f(x)$ fonksiyonunun $(n+1)$-inci mertebeden türevinin bu sayıdaki değeridir. Fakat, genelde $\xi_{x}$ sayısı tam olarak bilinmez ve bu durumda hata hakkında bir tahmin yapılmasını sağlayan aşağıdaki hata formülü önemlidir [2]:

$$
\begin{aligned}
\max _{x \in[a, b]}\left|f(x)-p_{n}(x)\right| \\
\leq \max _{x \in[a, b]}\left(\frac{\left|f^{(n+1)}(x)\right|}{(n+1) !}\right) \max _{x \in[a, b]} \prod_{j=0}^{n}\left|x-x_{j}\right|
\end{aligned}
$$

Burada, $f(x)$ fonksiyonu problem tarafindan sabitlenmiş olduğundan hatanın üst sınırı üzerinde meydana gelebilecek herhangi bir etki; Denklem (8)'deki Cauchy hata çarpanı olarak 
adlandırılan $Q(x)=\prod_{j=0}^{n}\left(x-x_{j}\right)$ ifadesinden dolayı, sadece $x_{j}$ noktalarının seçimine bağlı olacaktır. İnterpolasyon noktaları $(n+1)$-inci dereceden Chebyshev polinomunun kökleri olacak şekilde seçilirse, $\max _{x \in[-1,-1]} \prod_{j=0}^{n}\left|x-x_{j}\right|$ ifadesi minimum olur ve bu değer $\frac{1}{2^{n}}$ 'dir [2]. Bu noktalara Chebyshev noktaları denir ve

$$
x_{j}=\cos \left(\frac{2 j+1}{2 n+2} \pi\right), j=0, \cdots, n
$$

şeklinde tanımlanırlar. Cauchy hata çarpanının mutlak değerinin maksimum değerini $\frac{1}{2^{n-1}}$ ile minimuma yakın yapan bir başka noktalar kümesi ise Chebyshev polinomunun ektremumları olarak adlandırılan ve tanım aralığının uç noktaları olan \pm 1 sayılarını içermeleriyle Chebyshev noktalarından ayrışan Chebyshev-Lobatto noktalarıdır ve bu noktalar aşağıdaki şekilde tanımlanır:

$$
x_{j}=\cos \left(\frac{j}{n} \pi\right), j=0, \cdots, n .
$$

Eğer noktalar eşit aralıklı olarak,

$$
x_{j}=-1+\frac{2 j}{n}, j=0, \cdots, n
$$

şeklinde seçilirse, Chebyshev-Lobatto noktalarının aksine $\max _{x \in[-1,1]}|Q(x)|$ ifadesi $\frac{(2 n) !}{2^{n} n^{n+1} n !}$ gibi büyük değerli bir alt sınır ile kötü sonuç verecektir.

\section{Mock-Chebyshev İnterpolasyonu}

İnterpolasyon polinomunun eşit aralıklı noktalardaki davranışları ile Chebyshev noktalarındaki davranışları kıyaslandığında çok farklı sonuçlar verdiği görülmektedir. Dahası, Chebyshev noktaları optimale yakın sonuç verirken eşit aralıklı noktalar ise, Runge olgusundan dolayı, analitik fonksiyonlar için dahi rraksak sonuç verebilmektedir. İyi bir yaklaşım elde etmek için izlenecek yol, polinom interpolasyonunda asimtotik olarak Chebyshev noktalarının dağılımını takip eden noktalar kümesinin kullanılmasıdır [8]. Fakat, bir gözlem veya ölçüm sırasında veriler genelde eşit aralıklı olacak şekilde elde edilir. Bu durumda, eşit aralıklı nokta dağılımının kötü sonuçlarından kaçınmak ve polinom interpolasyonunun hatasını azaltmak için son yıllarda geliştirilen mock-Chebyshev noktaları olarak adlandırılan iyi bir alternatif yöntem vardır (Şekil 1 ve Şekil 2). Bu yöntem, polinom interpolasyonunda kullanılacak verilerin eşit aralıklı yeteri kadar geniş̧̧e bir kümesinden Chebyshev-Lobatto noktalarının dağılımı dikkate alınarak bir alt küme oluşturulmasına dayanır. $\mathrm{Bu}$ küme oluşturulurken elde edilen noktaların aşağıdaki iki koşulu sağlaması gerekir:

(i) Noktalar biririnden faklı olmalıdır.

(ii) Noktaların dağılımı Chebyshev-Lobatto noktalarının

dağılımına benzer olmalıdır.

$\mathrm{Bu}$ iki koşulu sağlayan noktalar kümesinin özellikleri yakın zamanda yayınlanan bir çalışmada [6] gösterilmiştir.



Şekil 1. Runge fonksiyonu (kesikli çizgi) ve 11 eşit aralıklı noktada fonksiyonun interpolasyon polinomu (düz çizgi)

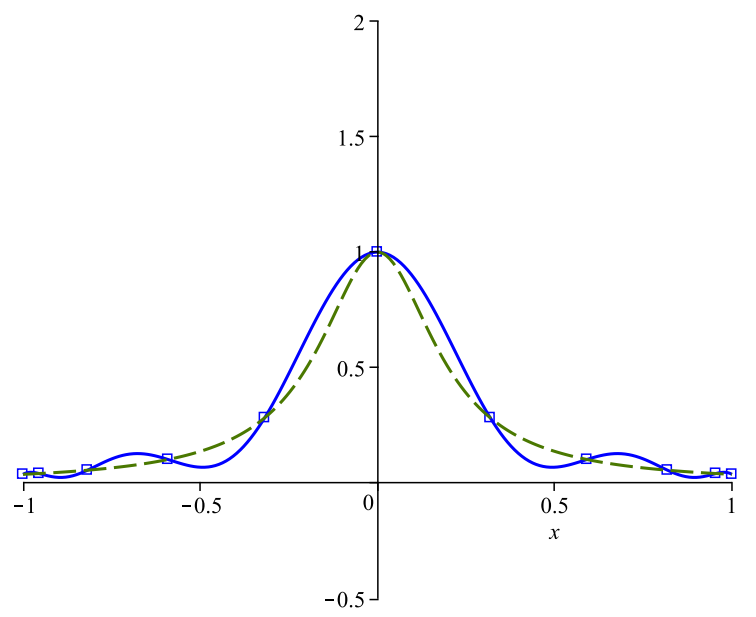

Şekil 2. Runge fonksiyonu (kesikli çizgi) ve 11 mock-Chebyshev noktasında fonksiyonun interpolasyon polinomu (düz çizgi) 


\subsection{Mock-Chebyshev Noktalarının Hesabı}

Mock-Chebyshev noktalarının nasıl seçileceği konusunda bir yöntem ilk kez Boyd [1] tarafindan makalesinde verilmiştir. Fakat, bu yöntem lokal optimizasyon gerektirdiğinden $O\left(n^{3}\right)$ mertebeden işlem yükü ile maliyeti yüksektir. Ancak, bu işlem yükünü $O\left(n^{2}\right)$ mertebesine indiren aşağıdaki yöntem, mock-Chebyshev noktalarının elde edilmesinde iyi bir alternatif sunmaktadır. $\mathrm{Bu}$ yöntemde, $(n+1)$ adet $\tilde{x}_{0}, \tilde{x}_{1}, \ldots, \tilde{x}_{n}$ mockChebyshev noktası $m \geq\left\lceil\frac{2 n^{2}}{\pi^{2}}\right\rceil+1$ olmak üzere $(m+1)$ adet $\bar{x}_{0}, \bar{x}_{1}, \ldots, \bar{x}_{m}$ eşit aralıklı noktalar arasından aşağıdaki gibi seçilirler.

$$
\begin{aligned}
& X_{n}=\left\{x_{j}=-\cos \left(\frac{j \pi}{n}\right), \quad j=0,1, \ldots, n\right\}, \\
& \bar{X}_{m}=\left\{\bar{x}_{k}=-1+\frac{2 k}{m}, \quad k=0,1, \ldots, m, m \geq\left\lceil\frac{2 n^{2}}{\pi^{2}}\right\rceil+1\right\}, \\
& \tilde{X}_{n}=\left\{\tilde{x}_{j}: \tilde{x}_{j} \in \bar{X}_{m}, \tilde{x}_{j} \approx x_{j}, j=0,1, \ldots, n\right\},
\end{aligned}
$$

olmak üzere, $\tilde{X}_{n}$ kümesinin elemanları aşağıdaki şekilde oluşturulur.

\section{Metod 1:}

$\bar{X}_{m, 0}=\left\{\bar{x}_{0}=-1\right\}, \bar{X}_{m, n}=\left\{\bar{x}_{n}=1\right\}$,

$\bar{X}_{m, j}=\left\{\bar{x}_{l}: \frac{x_{j-1}+x_{j}}{2}<\bar{x}_{l}<\frac{x_{j}+x_{j+1}}{2}, 1 \leq j \leq n-1, l \in\{0,1, \ldots, m\}\right\}$,

$\tilde{x}_{j} \in \bar{X}_{m, j}, j=0,1, \ldots, n$.

Bu yöntemin avantajlarından biri, $\tilde{x}_{j} \in \bar{X}_{m, j}, j=0,1, \ldots, n$

seçimine bağlı olarak birden fazla kümenin elde edilebilmesidir. Elde edilen her bir küme yukarıda belirtilen mock-Chebyshev noktalar kümesi olma koşullarını sağlar. Örneğin,

$\forall \bar{x}_{l} \in \bar{X}_{m, j}$ için $\left|x_{j}-\bar{x}_{l}\right|$ minimum ise, $\tilde{x}_{j}=\bar{x}_{l}$

seçimi ile noktalar arasındaki maksimum sapma dikkate alındığında en iyi,

$$
\forall \bar{x}_{l} \in \bar{X}_{m, j} \operatorname{için}\left|x_{j}-\bar{x}_{l}\right| \text { maksimum ise, } \tilde{x}_{j}=\bar{x}_{l}
$$

seçimi ile de en kötü mock-Chebyshev noktalar kümesi elde edilir. Buradaki en iyi ve en kötü olma durumuna göre oluşan noktaların kümelerini sırasıyla $\tilde{X}_{n}^{B}$ ve $\tilde{X}_{n}^{W}$ ile göstereceğiz. $\mathrm{Bu}$ yöntemin bir başka avantajı ise her bir seçimde işlem yükünün $O\left(n^{2}\right)$ mertebesinde olmasıdır. Bu bağlamda, sadece en iyi mock-Chebyshev noktalar kümesini veren fakat işlem yükü $O\left(n^{3}\right)$ mertebesinde olan bir yöntem Boyd [1] tarafindan verilmiştir. Bu yöntem aşağıdaki gibidir.

\section{Metod 2 [1]:}

$$
\begin{aligned}
& \left|x_{j}-\bar{x}_{l}\right|=\min _{k}\left|x_{j}-\bar{x}_{k}\right| \text { olacak şekilde } \tilde{x}_{j}=\bar{x}_{l}, \\
& \forall k=0,1, \ldots, m, \quad j=0,1, \ldots, n, \quad l \in\{0,1, \ldots, m\}
\end{aligned}
$$

$\mathrm{Bu}$ yöntemlere ilaveten, eşit aralıklı noktaların sayısının serbestçe seçilebildiği durumda, $O(n)$ mertebeden işlem yükü ile mock-Chebysev noktalarını hesaplayan hızlı bir algoritma yakın zamanda yayınlanan bir çalışmada [4] ve bunun genişletilmiş bir versiyonu da daha sonraki bir çalışmada [5] verilmiştir.

\section{Metod 3 [4]:}

$$
\begin{aligned}
& \tilde{x}_{j}=\tilde{x}_{0}+S_{j} \tilde{h}, \tilde{h}=\frac{2}{S_{n}}, \tilde{x}_{0}=x_{0}, S_{0}=0, j=1,2, \ldots, n . \\
& S_{j}=\left\lceil\frac{h_{j}\left(X_{n}\right)}{\min h_{j}\left(X_{n}\right)}\right\rceil+S_{j-1}, h_{j}\left(X_{n}\right)=x_{j}-x_{j-1} .
\end{aligned}
$$

\section{Metod 4[5]:}

Algoritma 1: $X_{n}$ kümesini oluşturma yordamı

girdi $X_{n}=\left\{x_{j}=\frac{1}{2}(a+b)+\frac{1}{2}(a-b) \cos \left(\frac{j \pi}{n}\right), \quad j=0,1, \ldots, n\right\}$

$j=1$ den $n$ yedöngü

$\mid h_{j}\left(X_{n}\right) \leftarrow x_{j}-x_{j-1}$;

döngü sonu

$h_{\min }\left(X_{n}\right) \leftarrow \min _{1 \leq j \leq n} h_{j}\left(X_{n}\right)$;

$S_{0} \leftarrow 0$

$j=1$ den $n$ yedöngü

$\mid S_{j} \leftarrow\left\lceil\frac{h_{j}\left(X_{n}\right)}{h_{\min }\left(X_{n}\right)}\right\rceil+S_{j-1}$

döngü sonu

$\tilde{h} \leftarrow \frac{b-a}{S_{n}} ; \tilde{x}_{0} \leftarrow x_{0} ; \tilde{X}_{n} \leftarrow\left\{\tilde{x}_{0}\right\} ;$

$j=1$ den $n$ yedöngü

$\mid \begin{aligned} & \tilde{x}_{j} \leftarrow \tilde{x}_{0}+S_{j} \tilde{h} \\ & \tilde{X}_{n} \leftarrow \tilde{X}_{n} \cup\left\{\tilde{x}_{j}\right\}\end{aligned}$

\section{döngü sonu}

çıtı $\tilde{X}_{n}=\left\{\tilde{x}_{j}: j=0,1, \ldots, n\right\}$ 


\section{Sayısal Sonuçlar ve Tartışma}

Bu bölümde, Metod 1 ile elde ettiğimiz noktaların performansını ölçmek için 2020 yılında meydana gelen koronovirüs pandemisinde Türkiye'nin 18 Haziran 2020 ile 10 Eylül 2021 arası Worldometers [9]'in verilerine göre, doğrulanmış günlük koronavirüs vaka sayılarını kullanarak polinom interpolasyonları elde edelim. Bunun için, öncelikle, bu tarihler arasındaki 85 günlük veri setinden 15 tanesini seçerek interpolasyon polinomu ile bir yaklaşım elde edip sonra elde ettiğimiz polinom ile kullanmadığımız günlerin verilerini tahmin ederek yapılan hatayı hesaplayalım. $\mathrm{Bu}$ işlemleri hem $\tilde{X}_{n}^{B}$ hem de $\tilde{X}_{n}^{W}$ kümeleri için ayrı ayrı yapalım. Öncelikle, bu 15 günlük veriyi 85 günlük veri içerisinden 18 Haziran 2020 tarihini ilk gün, 10 Eylül 2021 tarihini ise 85 -inci gün olarak kabul edip ilk günden başlamak üzere 6'şar gün arayla eşit aralıklı olacak şekilde Tablo 1'de olduğu gibi seçelim.

Tablo 1. Verilerin eşit aralıklı olacak şekilde seçilmesi

\begin{tabular}{|c|c|c|c|}
\hline$j$ & $x_{j}$ & $f_{j}:$ vaka say1S1 & Tarih \\
\hline 0 & 1 & 5575 & 18 HAZIRAN 2021 \\
\hline 1 & 7 & 5703 & 24 HAZIRAN 2021 \\
\hline 2 & 13 & 5496 & 30 HAZIRAN 2021 \\
\hline 3 & 19 & 5299 & 6 TEMMUZ 2021 \\
\hline 4 & 25 & 5404 & 12 TEMMUZ 2021 \\
\hline 5 & 31 & 7680 & 18 TEMMUZ 2021 \\
\hline 6 & 37 & 12381 & 24 TEMMUZ 2021 \\
\hline 7 & 43 & 22083 & 30 TEMMUZ 2021 \\
\hline 8 & 49 & 24297 & 5 AĞUSTOS 2021 \\
\hline 9 & 55 & 27356 & 11 AĞUSTOS 2021 \\
\hline 10 & 61 & 21692 & 17 AĞUSTOS 2021 \\
\hline 11 & 67 & 18857 & 23 AĞUSTOS 2021 \\
\hline 12 & 73 & 17332 & 29 AĞUSTOS 2021 \\
\hline 13 & 79 & 20033 & 4 EYLÜL 2021 \\
\hline 14 & 85 & 23562 & 10 EYLÜL 2021 \\
\hline
\end{tabular}

Bu durumda,

$p_{14}\left(x_{j}\right)=f_{j}, j=0, \ldots, 14$

şeklinde interpolasyon şartlarını sağlayan $p(x)$ interpolasyon polinomu, monomial bazlar kullanılarak Denklem (3) veya Lagrange bazları kullanılarak Denklem (7) ile kolayca hesaplanabilir. $\mathrm{Bu}$ durumda elde edilen yaklaşım polinomu (kırmızı eğri) ve seçilen günler (sarı sütunlar) açık bir şekilde Şekil 2'de gösterilmiştir. Runge olgusu sebebiyle, grafiğin başlangıç ve bitiş noktaları civarında oldukça büyük sıçramalar görülmektedir. Burada oluşan hataların büyüklüklerini daha net bir şekilde Şekil 3'teki grafikte görmekteyiz. Buna göre, vaka tahmin sayılarının pozitif olduğu günler dikkate alındığında, en büyük hata 83-üncü günde oluşur. Dahası, gerçekte 23914 olan vaka sayısı bu yaklaşım ile 297303 olarak olarak hesaplanır.



Şekil 3. 85 günlük vakadan 15 tanesinin eşit aralıklı olarak seçilmesiyle elde edilen interpolasyon polinomu

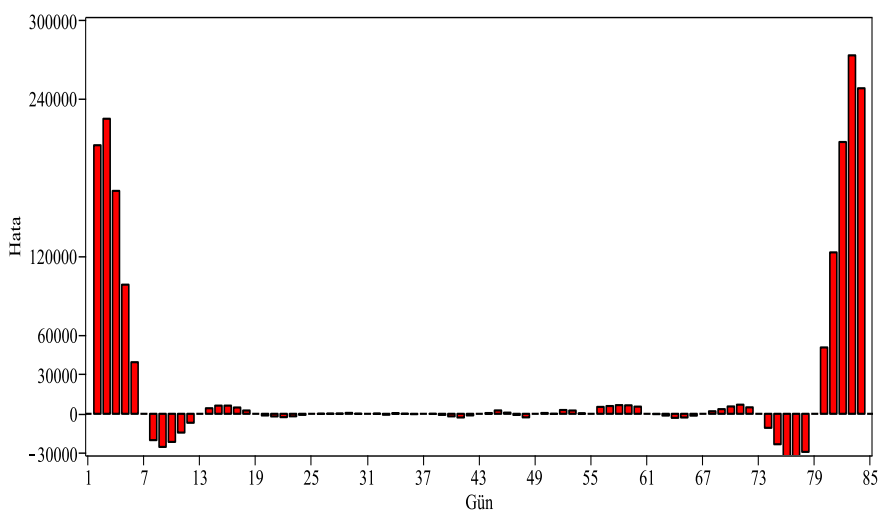

Şekil 4. Interpolasyon polinomu ile elde edilen dĕgerler ile gerçek değerler arasındaki farklar

Elde edilen yaklaşımın hatasını azaltmak için, şimdi ise, 15 günlük veriyi 85 günlük veri seti içerisinden mock-Chebyshev noktalarını dikkate alarak seçelim. Bunun için, Method 1 ile verileri önce $\tilde{X}_{14}^{B}$ ye göre Tablo 2' de olduğu gibi seçelim.

Tablo 2. Verilerin Metod 1 ile $\tilde{X}_{14}^{B}$ ye göre seçilmesi

\begin{tabular}{|c|c|c|c|}
\hline$j$ & $x_{j}$ & $f_{j}:$ vaka say1s1 & Tarih \\
\hline 0 & 1 & 5575 & 18 HAZIRAN 2021 \\
\hline 1 & 2 & 5480 & 19 HAZIRAN 2021 \\
\hline 2 & 5 & 6143 & 22 HAZIRAN 2021 \\
\hline 3 & 10 & 4883 & 27 HAZIRAN 2021 \\
\hline 4 & 17 & 4418 & 4 TEMMUZ 2021 \\
\hline 5 & 25 & 5404 & 12 TEMMUZ 2021 \\
\hline 6 & 34 & 8151 & 21 TEMMUZ 2021 \\
\hline 7 & 43 & 22083 & 30 TEMMUZ 2021 \\
\hline 8 & 52 & 22699 & 8 AĞUSTOS 2021 \\
\hline 9 & 61 & 21692 & 17 AĞUSTOS 2021 \\
\hline 10 & 69 & 19970 & 25 AĞUSTOS 2021 \\
\hline 11 & 76 & 23946 & 1 EYLÜL 2021 \\
\hline 12 & 81 & 20962 & 6 EYLÜL 2021 \\
\hline 13 & 84 & 23846 & 9 EYLÜL 2021 \\
\hline 14 & 85 & 23562 & 10 EYLÜL 2021 \\
\hline
\end{tabular}


$\mathrm{Bu}$ durumda, seçilen günler ve elde edilen interpolasyon polinomu Şekil 4'teki, oluşan interpolasyon hataları ise Şekil 5 'teki gibidir. $\mathrm{Bu}$ grafikler incelendiğinde, eşit aralıklı seçime göre oluşan hatanın büyük ölçüde azaldığı görülür. Dahası, en büyük hatanın 73-üncü günde oluştuğu yani gerçekte 17332 vaka varken bu yaklaşım ile 22583 olarak tahmin edileceği görülür.

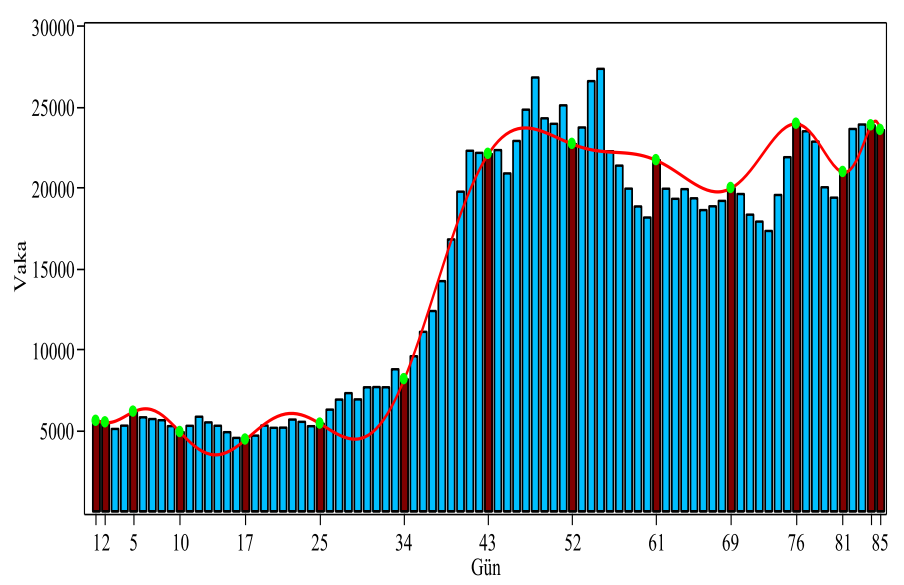

Şekil 5. Verilerin Metod 1 ile $\tilde{X}_{14}^{B}$ ye göre seçilmesiyle elde edilen interpolasyon polinomu

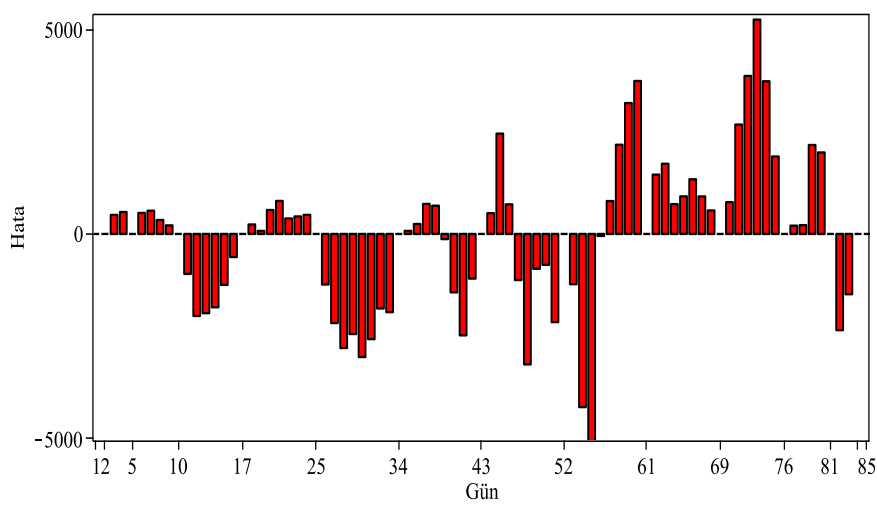

Şekil 6. Interpolasyon polinomu ile elde edilen değerler ile gerçek değerler arasındaki farklar

Şimdi ise, yine Method 1 ile verileri $\quad \tilde{X}_{14}^{W}$ ye göre Tablo 3' te olduğu gibi seçelim.

Tablo 3. Verilerin Metod 1 ile $\tilde{X}_{14}^{W}$ ye göre seçilmesi

\begin{tabular}{|c|c|c|c|}
\hline$j$ & $x_{j}$ & $f_{j}:$ vaka sayıs1 & Tarih \\
\hline 0 & 1 & 5575 & 18 HAZIRAN 2021 \\
\hline 1 & 3 & 5091 & 20 HAZIRAN 2021 \\
\hline 2 & 7 & 5703 & 24 HAZIRAN 2021 \\
\hline 3 & 13 & 5496 & 30 HAZIRAN 2021 \\
\hline 4 & 20 & 5160 & 7 TEMMUZ 2021 \\
\hline 5 & 29 & 6918 & 16 TEMMUZ 2021 \\
\hline 6 & 38 & 14230 & 25 TEMMUZ 2021 \\
\hline 7 & 39 & 16809 & 26 TEMMUZ 2021 \\
\hline
\end{tabular}

\begin{tabular}{|c|c|c|c|}
\hline 8 & 48 & 26822 & 4 AĞUSTOS 2021 \\
\hline 9 & 57 & 21372 & 13 AĞUSTOS 2021 \\
\hline 10 & 66 & 18622 & 22 AĞUSTOS 2021 \\
\hline 11 & 73 & 17332 & 29 AĞUSTOS 2021 \\
\hline 12 & 79 & 20033 & 4 EYLÜL 2021 \\
\hline 13 & 83 & 23914 & 8 EYLÜL 2021 \\
\hline 14 & 85 & 23562 & 10 EYLÜL 2021 \\
\hline
\end{tabular}

$\mathrm{Bu}$ durumda, seçilen günler ve elde edilen interpolasyon polinomu Şekil 6'daki, oluşan interpolasyon hataları ise Şekil 7'deki gibidir. Bu seçimde, en büyük hatanın 45-inci günde oluştuğu yani gerçekte 20890 vaka varken bu yaklaşım ile 27452 olarak tahmin edileceği görülür. Burada verilerin mockChebyshev noktalarının en kötü seçimi olan $\tilde{X}_{14}^{W}$ ye göre seçilse bile eşit aralıklı seçim ile karşılaştırıldığında oluşan hatanın büyük ölçüde azaldığı görülür.



Şekil 7. Verilerin Metod 1 ile $\tilde{X}_{14}^{W}$ ye göre seçilmesiyle elde edilen interpolasyon polinomu

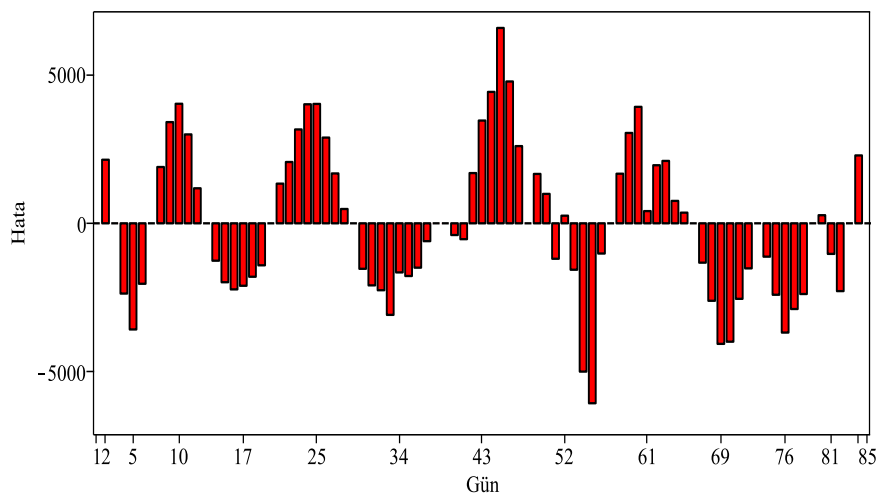

Şekil 8. Interpolasyon polinomu ile elde edilen değerler ile gerçek değerler arasındaki farklar

Başka bir açıdan, bu 85 günlük zaman diliminde her gün değil de sadece 15 gün seçilip bu günlerde test yapılmış ve kalan diğer 70 günlük vakalar ise tahmin edilmek istenseydi, eşit aralıklı gün seçimine göre yapılacak maksimum bağıl hatanın büyüklüğü mock-Chebyshev noktalarının en kötü seçimi olan $\tilde{X}_{14}^{W}$ ye göre seçilse bile ile elde edilenin yaklaşık 36 katı olacaktı. 


\section{Sonuç}

$\mathrm{Bu}$ çalışmada, polinom interpolasyonda eşit aralıklı noktaların kötü sonuçlarından kaçınmak için önerilen mockChebysev noktalarını daha az işlem yükü ile hesaplayan bir yöntem verildi. Dahası, bu yöntem ile mock-Chebyshev noktalarının birbirinden farklı kümelerinin elde edilebileceği gösterildi. $\mathrm{Bu}$ yöntem ile elde edilen noktalara karşılık gelen günlerdeki veriler kullanılarak, Kovid-19 vakalarını ara günlerde tahmin etmek için interpolasyon polinomları elde edildi. Bulunan sonuçlar, günlerin eşit aralıklı olacak şekilde seçilmesi durumunda elde edilecek sonuçlar ile karşılaştırıldı. $\mathrm{Bu}$ karşılaştırmada, eşit aralıklı günler için çok büyük interpolasyon hatası elde edilirken, önerilen metod ile elde edilen günler kullanıldığında ise en kötü seçimde bile polinom interpolasyonda optimale yakın sonuç veren Chebysev noktalarına benzer şekilde, bu hatanın oldukça az olduğu görüldü.

\section{Kaynakça}

[1] Boyd, J. P., \& Xu, F. (2009). Divergence (Runge phenomenon) for least-squares polynomial approximation on an equispaced grid and Mock-Chebyshev subset interpolation. Applied Mathematics and Computation, 210(1), $158-168$.

[2] Davis, P.J. (1963). Interpolation and Approximation, Blaisdell Publishing Co. Ginn and Co., New York.

[3] Ibrahimoglu, B.A. (2016). Lebesgue functions and lebesgue constants in polynomial interpolation, J. Inequal. Appl. 2016 (1) 93.

[4] Ibrahimoglu, B.A. (2020). A fast algorithm for computing the mock-Chebyshev nodes, J. Comput. Appl. Math. Vol.373, 112336 .

[5] İbrahimoğlu, B. A. (2021). Mock-Cebyshev Noktalarında Vandermonde Matrisinin Bir Uygulamasi: Kovid-19 Vaka Tahmini . Avrupa Bilim ve Teknoloji Dergisi , (21), 172-180.

[6] Ibrahimoglu, B.A. (2021). A new approach for constructing mock-Chebyshev grids, Math. Meth. Appl. Sci.

[7] Runge, C. (1901). Uber empirische Funktionen und die Interpolation zwischen aquidistanten Ordinaten, Z. Math. Phys., 46, 224-243.

[8] Trefethen, L.N. (2000). Spectral Methods in MATLAB, SIAM, Philadelphia.

[9] https://www.worldometers.info/coronavirus/country/turkey/ 